Germ warfare:

Researchers turn to genomics to tackle

infectious diseases

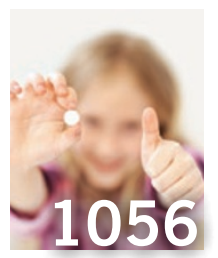

Kids' stuff:

Clinical trials with

children raise unique

ethical concerns

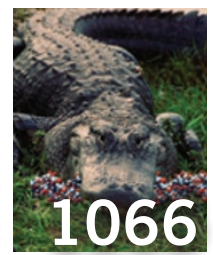

Sunshine biology:

Florida strives to

be the next

biopharma hub

\title{
Pooled studies can raise misleading alarm bells, regulators say
}

Two years ago, drug safety researchers released a critical report linking certain inhaled lung medications, including the blockbuster drug Spiriva, to elevated risks of heart attack, stroke and death from heart disease by more than $50 \%$ compared with other inhaled lung drugs or placebo treatment. Notably, the scientists reached this conclusion by pooling the results of 17 clinical trials, all of which tested the inhaled drugs for at least 30 days.

Safety concerns arising from similar metaanalyses have been sufficient to get notable top-selling drugs, including Merck's Vioxx and Novartis's Zelnorm, yanked from the pharmacy shelf. And the US Food and Drug Administration (FDA) came down quite harshly on GlaxoSmithKline's Avandia after a 2007 meta-analysis raised red flags. Yet, in January, the agency ruled that Spiriva, marketed by Pfizer and Boehringer Ingelheim, does not pose a significant health risk.

Without clear guidelines on how the FDA interprets meta-analyses that raise safety concerns, some are calling for increased transparency in the agency's decision making process. "The FDA as a whole has been inconsistent," says Sonal Singh, a pharmacoepidemiologist now at the Johns Hopkins Bloomberg School of Public Health in Baltimore who led the meta-analysis of the lung disease medications (J. Am. Med. Assoc. 300, 1439-1450, 2008). “They haven't put down the threshold to pull a drug. When they say a drug is safe, what do they mean?"

Clarifying its position, the FDA last month explained its stance on Spiriva and laid out general criticisms of studies that combine the results of multiple clinical trials to raise safety concerns. In an opinion article in the New England Journal of Medicine (363, 1097 1099, 2010), three FDA officials argued that such conglomerated studies often result in "urgent calls" to take action even when there are weaknesses in the analysis. "We must use measured restraint during our evaluations to ensure that safe drugs remain on the market," the authors wrote.

Many academics share the FDA's trepidations about relying too heavily on conclusions drawn from meta-analyses. With

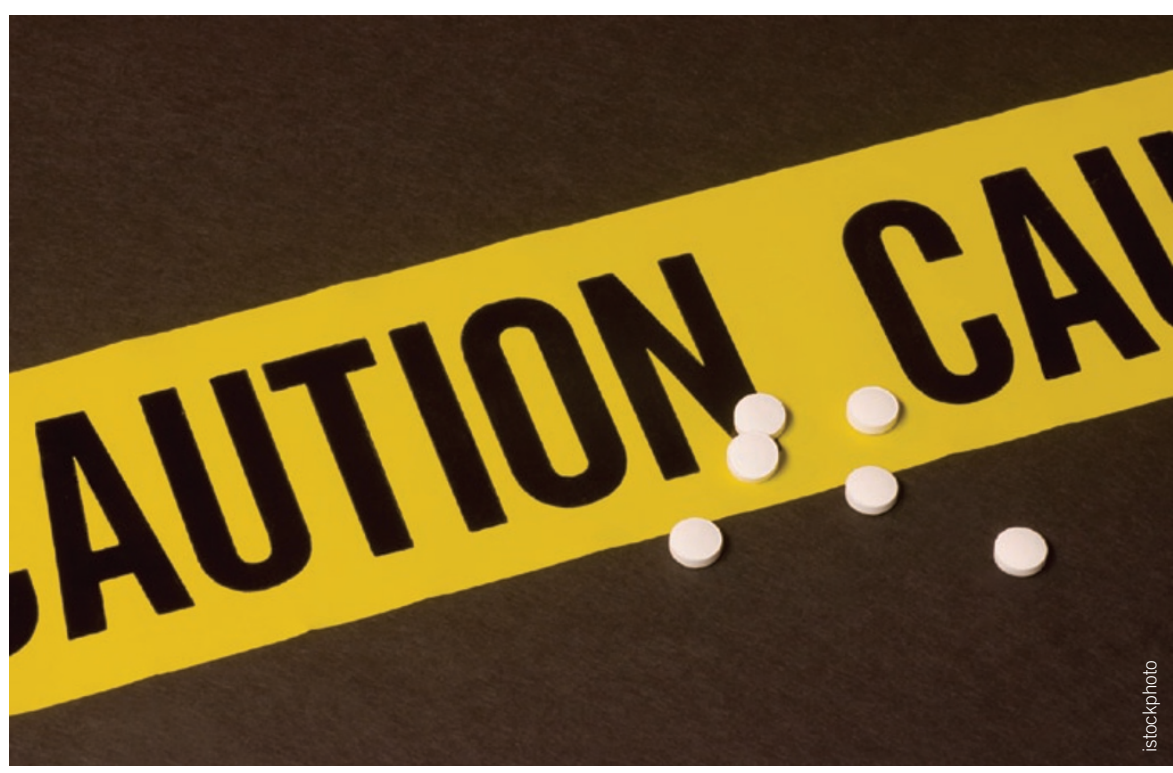

Cautionary tale: Meta-analyses can have flawed methods.

more than 4,000 such studies now written each year, compared to just 400 published annually 20 years ago, Peter Honig, head of global regulatory affairs for AstraZeneca and past founder and director of the FDA's Office of Drug Safety, worries about the robustness of these pooled investigations. "My concern is they are not being done by learned and skilled analysts," he says. "I am positive the quality is not uniform."

In an effort to develop standard practices, an international group led by David Moher from the Ottawa Hospital Research Institute recently published a 27 -item checklist and flow chart to help authors improve their meta-analyses (Ann. Intern. Med. 151, 264-269, 2009). "Most systematic reviews and meta-analyses are inadequately reported, and that can lead to bias in how they are interpreted," Moher says. "So we need to develop some techniques and interventions to improve the quality of the reporting of research."

For readers who would rather not follow a recipe, there are a few ways to gauge the validity of a meta-analysis. First, as in any scientific study, the hypothesis needs to be well defined, says Honig. "Say what you're going to do, and then do what you said you were going to do," he stresses. Second, check to see if the list of authors includes people appropriate for the task, including a researcher or clinician with subject matter knowledge and a methodologist who understands how methods differ from trial to trial. Third-"and this is one of the biggest mistakes made," according to Kay Dickersin, director of the US Cochrane Center, a not-for-profit organization that specializes in healthcare analysis and systematic reviewscheck where the authors got their evidence: clinical trials that can be found the most quickly in literature searches are more likely to have positive results, whereas negative results are sometimes buried in obscure journals or published in other languages. "It's very important to look deeply," Dickersin says.

In an ideal world, post-marketing drug safety concerns would always be tested with large, prospective, randomized clinical trials. But, considering the prohibitive expenses involved, Singh advocates keeping metaanalyses as a key diagnostic of drug safety. "We should be doing this for every drug on the market," he says.

Megan Scudellari 